\title{
LA ESCALA DREEM PARA MEDIR EL AMBIENTE EDUCACIONAL: ¿VALIDACIÓN TRANSCULTURAL, ACTUALIZACIÓN O AMBAS?
}

The DREEM scale to measure the educational environment: cross-cultural validation, update or both? Marco Pérez-Valencia ${ }^{1}$, Dayvid Canchari-Huamán ${ }^{1}$, Carlos Uría-Guerrero ${ }^{1}$, Percy Mayta-Tristán ${ }^{1}$

1. Escuela de Medicina, Universidad Peruana de Ciencias Aplicadas. Lima, Perú. Email de Contacto: carlosuriaguerrero@gmail.com

Señor Editor:

El ambiente educacional está definido por todas las características, intrínsecas (propios del alumno) y extrínsecas (propios de la universidad), que forman parte de la educación y puede ser evaluada a través de la percepción de los estudiantes. Así pues, existen diferentes instrumentos para analizarlo: Dundee Ready Educational Environment Measure (DREEM), Postgraduate Hospital Educational Environment Measure (PHEEM) y Clinical Learning Environment Scale (CLE). Dentro de estos, la escala DREEM ha sido verificada, como la más fidedigna. Ha sido validada en inglés, como idioma original, y aplicada en diferentes países y contextos culturales, demostrando consistencia y confiabilidad ${ }^{1}$. Además, a diferencia de otras, puede ser aplicada en alumnos de pre y post-grado de diferentes carreras de la salud. Está divida en cinco dominios (percepción del aprendizaje, percepción del docente, percepción académica, percepción de la atmósfera y percepción social) que permiten obtener resultados fiables y precisos puesto que analiza el ambiente educacional de manera integral ${ }^{2}$.

Con el objetivo de optimizar la educación, en diversas instituciones alrededor del mundo se ha validado y aplicado el DREEM. Sin embargo, no en todas se ejecutaron los procedimientos de manera correcta ${ }^{3}$. Para que un instrumento cuente con características apropiadas de aplicación (fiabilidad, sencillez, viabilidad, capacidad de medir sin error, entre otras), es necesario que sea sometido a un proceso de validación. Este tiene dos fases, la traducción y adaptación cultural son parte de la primera, y la evaluación de la validez y fiabilidad son parte de la segunda. La no aplicación de esta metodología genera resultados poco fiables y confusos, limita su interpretación y lo aleja de su objetivo principal ${ }^{4}$.

Existen estudios que han aplicado correctamente este proceso para la utilización del DREEM, mostrando variación tanto en el número y distribución de ítems como en la denominación y número de dominios luego de aplicar el análisis factorial confirmatorio y verificar que no tenían similar estructura (Tabla 1). 


\begin{tabular}{|c|c|c|c|c|c|c|c|}
\hline \multicolumn{8}{|c|}{ Tabla 1. Diferencias en la distribución de ítems y dominios en las diferentes versiones validadas del DREEM. } \\
\hline Estudio & Madificación & $\begin{array}{l}N^{\circ} \mathrm{de} \\
\text { ittems }\end{array}$ & Daminio I & Daminio II & Daminio III & Daminio IV & Daminio V \\
\hline $\begin{array}{l}\text { ORIGINAL }{ }^{2} \\
1997\end{array}$ & $\begin{array}{l}\text { Versíín } \\
\text { ariginalal }\end{array}$ & 50 & $\begin{array}{l}\text { Students' perception of } \\
\text { learning. Items }(n=12): 1,7,13 \text {, } \\
\text { IG, 20, 22, 24, 25, 38, 44, 47y } \\
48\end{array}$ & $\begin{array}{l}\text { Students' perception of } \\
\text { teachers. Items (n=II): 2, } \\
\text { 6, 8, }, \text {, 18, 29, 32, 37, 39, } \\
\text { 40 y 50 }\end{array}$ & $\begin{array}{l}\text { Students' academic self- } \\
\text { perceptions. Items(n=8): } \\
\text { 5, 10, 21, 26, 27, 31, } 41 \text { y } 45\end{array}$ & $\begin{array}{l}\text { Students' perceptions } \\
\text { of atmosphere. Items } \\
\text { (n=I2): II, 12, 17, 23, 30, } \\
33,34,35,36,42,43 \text { y } \\
49\end{array}$ & $\begin{array}{l}\text { Students' } \\
\text { social self- } \\
\text { perception. } \\
\text { Items (n=7) } \\
3,4,14,15, \\
19,28 \text { y } 46\end{array}$ \\
\hline $\begin{array}{l}\text { SUECIA(a) } \\
\text { 20II }\end{array}$ & $\begin{array}{l}\text { Se realiź́ el } \\
\text { análisis } \\
\text { factorial } \\
\text { confirmatori } \\
\text { a y } \\
\text { exploratorio. } \\
\text { Se modificó } \\
\text { la } \\
\text { denominació } \\
\text { n de } \\
\text { daminios y la } \\
\text { distribución } \\
\text { de ítems. }\end{array}$ & 50 & $\begin{array}{l}\text { Learning and mativation. } \\
\text { Items: I, 2, 3, 7, I2, I3, I4, I6, } \\
\text { 20, 21, 22, 24, 35, 40, 41, 43, } 44\end{array}$ & $\begin{array}{l}\text { Communication. Items: 6, } \\
\text { II, } 18,29,30,31,32,37\end{array}$ & $\begin{array}{l}\text { Psychasacial situation. } \\
\text { Items: 4, 15, 19, 23, 28, 33, } \\
\text { 34, 42, 45, 46 }\end{array}$ & $\begin{array}{l}\text { Teaching arganisation } \\
\text { and pragression. Items: } \\
5,10,26,27,36,38,47\end{array}$ & $\begin{array}{l}\text { Bad } \\
\text { teaching. } \\
\text { Items: } 8,9 \text {, } \\
17,25,39 \text {, } \\
48,49,50\end{array}$ \\
\hline $\begin{array}{l}\mathbb{I R A N}^{(b)} \\
2014\end{array}$ & $\begin{array}{l}\text { Se realizó un } \\
\text { análisis } \\
\text { factorial } \\
\text { confirmatori } \\
\text { o, se } \\
\text { modificó el } \\
\text { númera de } \\
\text { ítems }\end{array}$ & 44 & $\begin{array}{l}\text { Students' perception of } \\
\text { learning. Items: I, 7, 13, I6, } 20 \text {, } \\
24,25,38,44,47 \text { y } 48\end{array}$ & $\begin{array}{l}\text { Students' perception of } \\
\text { teachers. Items: } \\
\text { 2,6,8,8,18,29,32,37,40 y } 50\end{array}$ & $\begin{array}{l}\text { Students' academic self- } \\
\text { perceptions. Items: } \\
\text { 5,21,26,27,31,41 y } 45\end{array}$ & $\begin{array}{l}\text { Students' perceptions } \\
\text { of atmosphere. Items: } \\
11,12,23,30,33,34,35,36, \\
43 \text { y } 49\end{array}$ & $\begin{array}{l}\text { Students' } \\
\text { social self- } \\
\text { perception. } \\
\text { Items: } \\
\text { 3.,4,14.15,19,2 } \\
8 \text { y 46 }\end{array}$ \\
\hline $\begin{array}{l}\text { CHLLE }{ }^{\left({ }^{(}\right)} \\
2015\end{array}$ & $\begin{array}{l}\text { Se realizá el } \\
\text { análisis } \\
\text { factorial } \\
\text { confirmatori } \\
\text { a y } \\
\text { exploratorio. } \\
\text { Se eliminó un } \\
\text { domino y se } \\
\text { modificó la } \\
\text { distribución } \\
\text { y el número } \\
\text { de ítems }\end{array}$ & 40 & $\begin{array}{l}\text { Рeгcepción académica. Ítems: } \\
\text { 1, 2, 3, 7, 14, I6, 17, 20, 21, 22, } \\
\text { 24, 32, 37, 40, 41, 43, } 44 \text { y } 45\end{array}$ & $\begin{array}{l}\text { Experiencia académica. } \\
\text { Ittems: } \\
\text { 4,5,10,19,27,28,35,36 y } 42\end{array}$ & $\begin{array}{l}\text { Percepción de la } \\
\text { atmósfera. Ítems: } \\
\text { 6,II,I8,23,33,34 y } 49\end{array}$ & $\begin{array}{l}\text { Percepcíńn de los } \\
\text { docentes. Items: } \\
8,9,25,29,39 \text { y } 50\end{array}$ & \\
\hline $\begin{array}{l}\text { GHANA(d) } \\
\text { 2016 }\end{array}$ & $\begin{array}{l}\text { Se realizá el } \\
\text { análisis } \\
\text { factorial } \\
\text { confirmatori } \\
\text { a y } \\
\text { exploratorio. } \\
\text { Se modifićá } \\
\text { la } \\
\text { denominació } \\
\text { n y el } \\
\text { númera de } \\
\text { dominios, por } \\
\text { ende, la } \\
\text { distribución } \\
\text { de ítems. }\end{array}$ & 50 & $\begin{array}{l}\text { Perception of teachers, } \\
\text { learning and academic } \\
\text { atmasphere. Items: } \\
\text { 1,2,3,5,6,7,1,1,12,16,18, } \\
20,21,22,23,24,26,29,32,34,37 \text {, } \\
40\end{array}$ & $\begin{array}{l}\text { Motivation and } \\
\text { metacognition. Items: } \\
\text { 10,13,37,36,38,41,43,44,47, } \\
49\end{array}$ & $\begin{array}{l}\text { Perception of Social } \\
\text { environment. Items: } \\
\text { 14,15,19,28,30,31,33,427,45, } \\
\text { 46 }\end{array}$ & $\begin{array}{l}\text { Pour teaching and } \\
\text { learning strategies. } \\
\text { Items: } \\
4,8,9,17,25,35,37,48,50\end{array}$ & \\
\hline
\end{tabular}

(a) Jakobssan U, Danielsen N, Edgren G. Psychometric evaluation of the Dundee Ready Educational Enviranment Measure: Swedish version. Med Teach. 2011; 33: e267-e274.

(b) Koohpayehzadeh لJ, Hashemi A, Soltani K, Bigdeli S, Monsavi M, et al. Assessing validity and reliability of Dundee ready educational enviranment measure (DREEM) in Iran. Med J Islam Repub Iran. 2014; 28(C0): 1-9.

(c) Drtega ل, Pérez C, Drtiz L, Fasce E, McColl P, et al. Estructura factorial de la escala DREEM en estudiantes de medicina chilenas. Rev Med Chile. 2015; 143: 651-657.

(d) Mogre V. Amalba A. Psychometric Properties of the Dundee Ready Educational Enviranment Measure in a Sample of Chanaian Medical Students. Educ Health. 20I6; 29:16-24. 
Estas diferencias de estructura corroboran la importancia de la interpretación de resultados y de la adaptación cultural en cada país de aplicación, incluso ante la disponibilidad de una validación previa al idioma en el que se desea aplicar. Por otro lado, estas diferencias en la composición de los dominios y número de ítems puede reflejar también un cambio en el ambiente educacional debido al mayor uso de tecnologías de información y de la simulación clínica ${ }^{5}$ que no eran tan usadas al momento de la creación del instrumento, por lo que se debe revisar si se requiere una actualización del DREEM.

En conclusión, una correcta traducción y adaptación cultural, no garantiza la preservación de las propiedades psicométricas del instrumento original, por lo que es necesaria su validación al idioma y contexto cultural del país. Asimismo, ante la evidencia de los cambios estructurales realizados al DREEM en varios estudios, es importante verificar que este cuestionario con 20 años de antigüedad continúe siendo confiable pese al cambio conceptual que sufre constantemente el ambiente educacional.

\section{Bibliografía}

1. Soemantri D, Herrera C, Riquelme A. Measuring the educational environment in health professions studies: A systematic review. Med Teach. 2010;32(12):947-952.

2. Roff S, McAller S, Harden R, Al-Qahtani M, Ahmed A, et al. Development and validation of the Dundee Ready Education Environment Measure (DREEM). Med Teach. 1997;19(4):295-299.

3. Herrera C, Pacheco J, Rosso F, Cisterna C, Aichele D, et al. Evaluación del ambiente educacional pre-clínico en seis Escuelas de Medicina en Chile. Rev med Chile. 2010;138(6):677-84.

4. Ramada-Rodilla JM, Serra-Pujadas C, Delclós-Clanchet G. Adaptación cultural y validación de cuestionarios de salud: revisión y recomendaciones metodológicas. Salud Publica Mex. 2013;55(1):57-66.

5. Han H, Resch DS, Kovach RA. Educational Technology in medical education. Teach Learn Med. 2013;25(1):39-43. 